\title{
A Survey on Machine Learning and Text Processing for Pesticides and Fertilizer Prediction
}

\author{
P.Pandiaraja ${ }^{a}$, Lakshmi Priya T ${ }^{b}$, Pooja $D^{c}$, Prasath $M^{d}$, Swathi $D^{e}$ \\ Department of Computer Science and Engineering,M.Kumarasamy College of \\ Engineering, Karur, Tamil Nadu, India -639113 \\ b,c,d,e Department of Computer Science and Engineering, M.Kumarasamy College of Engineering, Karur, Tamil Nadu, India \\ $-639113$
}

Article History: Received: 11 January 2021; Accepted: 27 February 2021; Published online: 5 April 2021

\begin{abstract}
In the Agriculture sectors the pressure is increased immensely due to the rise in population. In this present year we mainly witness a move from conventional methods to the advanced technology with advent of the technology. Machine Learning and Text Processing have transformed the quality and the quantity of agriculture. The real time monitoring systems along with hybridization of species paved away for resource efficiency. Scientists and researchers across the globe are mainly shifting towards collaborative projects and ideologies to explore this field for saving society. The optimal solution has been provided by racing in the tech industry. This application will be portable, scalable and durable that provides help for initiating new areas in the agriculture field. So, this survey focuses on machine learning methodologies along with Text Processing systems in agriculture. This startup's private and public sector which is around the world that provides smart and sustainable solutions are briefly discussed. Based on agriculture this scenario, limitations, applications, development and future parameters are also briefly explained. The greatest challenge for the future agriculture lies in making agriculture research and extension more demand driven and client oriented. In addition, laboratory analysis has been undertaken to assess the nitrogen status and the soil carbon of fresh paddy soils and the used paddy soils as well as saplings. During the first season of 2011 the data collected on pests, disease incidence and severity and yield are being compiled for analysis. Therefore, integrated research to develop a bottom up participatory technology extension approach using new technologies is suggested for sustainable development in agriculture.
\end{abstract}

Keywords: Machine Learning, Text Processing, Random Forest Algorithm, Flutter Framework.

\section{Introduction}

Agriculture is one of the benchmark areas and milestone feelings of mankind. The word agriculture is found in the Latin language where ager is land and culture is cultivation [8]. According to the Food and Agricultural Organization of UN the humans depend on agriculture for their survival more than sixty percent and around twelve percent of the land is for the crop production. Presumed by the Food and Agricultural Organization, the whole native would make it eight billion by the year 2025 then ten billion by the end of 2050 [8]. To manage this growth in population an increase of seventy percent in crop production is estimated to achieve all over the globe by 2050. Agriculture has been ranked 2nd for emitting greenhouse, biomass, machinery and fossil based fertilizers and also it is the backbone of the economy for some of the nation [2]. Figure 1 shows the growth of the employment sector all over the world.

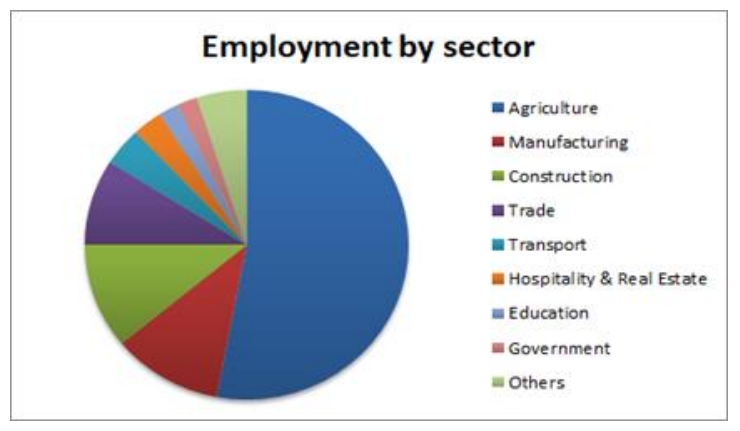

Figure 1: Employment by sector with GDP Growth (2016-2021)

Table 1. GDP Growth in BRICS \& G7

\begin{tabular}{|l|l|l|l|} 
& & & \\
Countries & 5 year Moving Average to 2016 & $\begin{array}{l}\text { 20 year Moving Average } \\
\text { to 2016 }\end{array}$ & $\begin{array}{l}\text { IMF Projected Annual Growth } \\
\text { 2016-2021 }\end{array}$ \\
\hline & & BRICS & \\
\hline
\end{tabular}


P.Pandiaraja ${ }^{a}$, Lakshmi Priya $T^{b}$, Pooja $D^{c}$, Prasath $M^{d}$, Swathi $D^{e}$

\begin{tabular}{|l|l|l|l|}
\hline Brazil & $-0.40 \%$ & $2.30 \%$ & $1.60 \%$ \\
\hline Russia & $0.50 \%$ & $3.30 \%$ & $1.50 \%$ \\
\hline India & $6.80 \%$ & $6.90 \%$ & $7.70 \%$ \\
\hline China & $7.30 \%$ & $9.20 \%$ & $6.10 \%$ \\
\hline South Africa & $1.60 \%$ & $2.80 \%$ & $1.80 \%$ \\
\hline & & $\mathbf{G 7}$ & \\
\hline US & $2.10 \%$ & $2.30 \%$ & $2.10 \%$ \\
\hline Japan & $1.20 \%$ & $0.70 \%$ & $0.70 \%$ \\
\hline Germany & $1.20 \%$ & $1.40 \%$ & $1.80 \%$ \\
\hline UK & $2.10 \%$ & $2.00 \%$ & $1.40 \%$ \\
\hline Canada & $1.80 \%$ & $1.90 \%$ & $1.90 \%$ \\
\hline
\end{tabular}

In India fifty three percent of the nation's natives are totally relying on agriculture for jobs and sixty two percent of the nation's natives are dependent essentially on agriculture for their survival [25]. By Focusing on the size of the market, India is the 2nd largest in producing the fruit industry across the globe. Based on the statistics, the income of farming in India is to be expected to double by the year 2022 [40]. Due to the increase in the demand with the rise of population, results are unstable in the coming years. Some of the important factors that should be taken into consideration are global warming and climate conditions [7]. According to the contributions of Machine Learning and its related technologies in the development and advancement of agricultural fields, this research has been done by going through some of the articles [4].

\section{Technical Backgrounds}

The main focus of this survey is how Machine Learning, Text Processing, Random Forest Algorithm and Flutter Framework are associated with this problem statement. In this section we have tried to bring overview and general purpose of Machine Learning, Text Processing, Random Forest Algorithm and Flutter Framework.

2.1 Machine Learning

Inspired by the various technologies of machine learning for connecting the globe, machine learning has been used everywhere from automating secular tests to offer the industries in all the sectors, intelligent insights that try to benefit them [9]. Machine Learning is defined as an algorithm which allows the applications to be accurate in predicting the future without externally or internally coded [36]. Basic task of machine learning is to make as many algorithms which can get input and use some of the mathematical analysis for predicting the result [1]. Machine Learning can be further divided into three types of learning 1.Supervised Learning 2.Unsupervised Learning 3.Reinforcement Learning. In Supervised learning, the machine is fed with labeled data where each data is tagged with the correct label. This is further classified into Classification and Regression techniques [26]. In Unsupervised learning, the machine is fed this uncategorized data and the machine will not be trained prior. This is further classified into Clustering and Association [47]. In Reinforcement learning, the machine will not be fed with any data instead it will interact with the environment. It receives rewards if it performs correctly and punishment if it fails to perform.

The Uses of Machine Learning in agriculture are 1.To boost the yield of crops which will determine the weather condition to give the highest return. 2. Agriculture robots are now programmed by many companies to manage harvesting crops and also work faster than then human laborers 3. Monitor crops, farmers can also find effective steps to save their crops and protect them from the weeds with the help of present technologies. 4 . The Retailers use present technologies to collect the data to create better crops and the pest control companies use them to identify various bacteria, bugs and diseases. 5. Pest control, the image recognition technology that identifies and treats various kinds of pests and vermin [16]. The various examples for important agricultural plants damaged by eating insect pests are highlighted in Table 2 .

Table 2: agricultural crops damaged by eating insect pests.

\begin{tabular}{|l|l|l|l|l|}
\hline Crops & Pests & Insect Order & $\begin{array}{l}\text { Actual Production of crop } \\
\text { in tons }\end{array}$ & $\begin{array}{l}\text { Crop damage by insect in } \\
\text { tons(approx) }\end{array}$ \\
\hline Rice & Nilaparvatalugens & Hemiptera & 96.7 & 32.2 \\
\hline Cotton & Helicoverpaarmigera & Lepidoptera & 44.03 & 18.9 \\
\hline Sugar cane & Scirpophaga novella & Lepidoptera & 348.2 & 87.1 \\
\hline
\end{tabular}




\begin{tabular}{|l|l|l|l|l|}
\hline Wheat & Sitobianavenae & Hemiptera & 78.6 & 4.1 \\
\hline Ground nut & Stomopterixnertaria & Lepidoptera & 9.5 & 1.7 \\
\hline
\end{tabular}

2.2 Random Forest Algorithm

The Random Forest algorithm which comes under supervised learning technique is one of the popular algorithms of ML. The Random Forest algorithm is used for classification and regression. [36]. The Random Forest Algorithm is mainly based on the concept of combining multiple classifiers which can solve a complex problem that improvises the performance of the model. This algorithm is also known as Ensemble Learning in machine learning [14]. Ensemble classification methods are based on constructing a group of classifiers instead of a single classifier which then classifies into new data values by taking a result of the predictions calculated before. These are classified as bagging, boosting and random forest [12]. In Random Forest Algorithm, the classifiers are defined as the group of tree-like classifiers. This is an advanced type of the technique that was classified from supervised learning and named as bagging in which rather than dividing each value using the best division among all the values, random forest divides each value by using the best one among a subset of values which are randomly selected at that value. Then using random feature selection a tree is built [14]. This strategy makes random forest excel in accuracy which when compared to other algorithms like Convolutional Neural Network; K means clustering and deep learning. Recent works of IOT projects for Crop Protection are highlighted in Table 3.

Table 3: Recent works on IOT on Crop Protection

\begin{tabular}{|c|c|c|}
\hline Reference of Proposed Works & Dedicated task of a Crop protection & Main Contributions \\
\hline $\begin{array}{l}\text { Harshani P } \quad \mathrm{R}, \quad \text { Umamaheswari } \\
\text { T,.Tharani R, Rajalakshmi S, and. } \\
\text { Dharani J[1] }\end{array}$ & $\begin{array}{l}\text { Effective crop production and nutrient } \\
\text { level monitor }\end{array}$ & $\begin{array}{l}\text { - Develop tools that analyse the } \\
\mathrm{pH} \text { of sands. }\end{array}$ \\
\hline Patil S S and Thorat S A[4] & Early detection of grapes diseases & $\begin{array}{l}\text { - Develop a system for } \\
\text { identifying grape diseases. }\end{array}$ \\
\hline Bauer $\mathrm{J}$ and Aschenbruck N[6] & $\begin{array}{l}\text { Implementation of an agricultural } \\
\text { monitoring system }\end{array}$ & $\begin{array}{l}\text { - The agricultural field is only } \\
\text { apparently refractory to the } \\
\text { digital technology and the } \\
\text { "smart farm" model is } \\
\text { increasingly widespread by } \\
\text { exploiting the Internet of } \\
\text { Things (IoT) paradigm } \\
\text { applied to environmental and } \\
\text { historical information through } \\
\text { time-series }\end{array}$ \\
\hline Coomans D, Massart D L[27] & $\begin{array}{l}\text { Supervised pattern recognition using } \\
\text { alternative voting rules }\end{array}$ & $\begin{array}{l}\text { - The performance criteria of } \\
\text { probabilistic methods are } \\
\text { discussed and the } \\
\text { performance of the condensed } \\
\text { nearest neighbour method is } \\
\text { compared with that of other } \\
\text { probabilistic pattern } \\
\text { recognition methods. }\end{array}$ \\
\hline
\end{tabular}

Various visual comparisons for the Random Forest algorithm for crop protection are highlighted in Figure 2. 


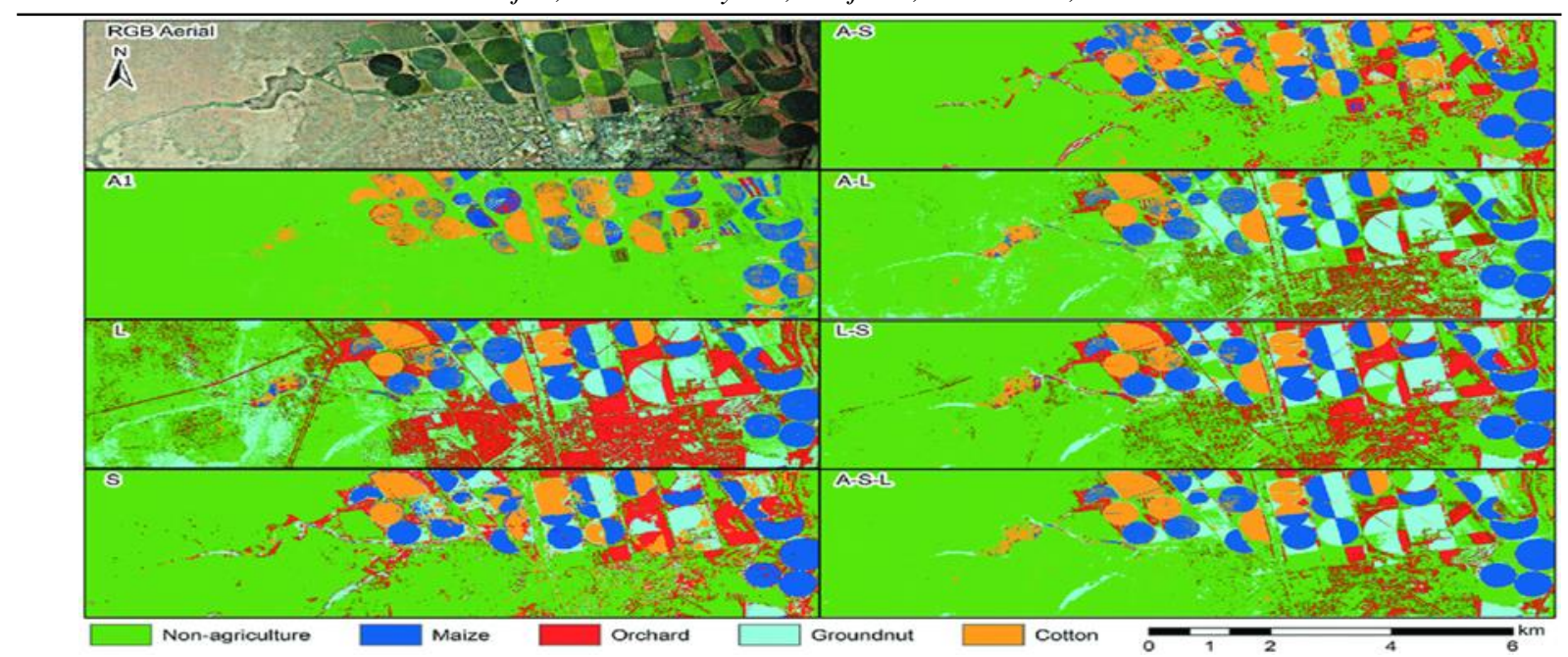

Figure 2. Visual comparison of the random forest algorithm

\subsection{Text Processing}

Natural Language Processing is one of the machine learning areas where a computer can understand, analyze and process the machine language that potentially converts into human language. It is used in information extraction, text simplification and spam filters in real life. NLP uses a natural language toolkit which is an open source package in python which provides all tools for common NLP tasks. The first task in NLP is preprocessing the data that is cleaning up the text data where the attributes are highlighted. Under preprocessing data the first step is to remove punctuation and then the text is separated into tokens which give structure to the previously unstructured text. Then the common words will be removed. After the preprocessing steaming is done. In steaming the suffices like "S", "ing" are removed by a simple approach. The next step is lemmatizing that derives the root form of the word the data is vectorized where the data is coded as integers.

2.4 Flutter Framework

Flutter is an open-source user interface framework and free of cost created by Google. It also allows us to create an android mobile app with only one code base where you can use only one programming language and only one code base to create any two different applications. There are two important parts in flutter which consists of a software development kit and a framework. SDK consists of tools that help in developing an application which also has different tools for compiling the code into object code. The Framework consists of a collection of reusable user interface elements where we can personalize our own needs. The Programming language which is used to develop applications with flutter is called Dart. Flutter has a set of customizable widgets to construct native interfaces in a few minutes. The Flutter is designed as an extensible, layered system. There will be no privileged access to any of the layers. The Flutter core is called a flutter engine which is commonly written in $\mathrm{c}++$ programming and also supports the primitives that are necessary to support all applications created by using flutter. The Flutter core provides the base-level implementations of Flutter's whole Application Programming Interface, including text layout, graphics, file, network input/output, plugin architecture, accessibility support, a dart RT and compiles tool chain. The Interaction is done by developers through the flutter framework with flutter that provides a modern, reactive framework which is coded in dart programming language. The Architecture of Flutter are highlighted in Figure 3.

\begin{tabular}{|c|c|c|c|}
\hline \multirow[t]{5}{*}{$\begin{array}{l}\text { Framework } \\
\text { Dart }\end{array}$} & Material & & Cupertino \\
\hline & \multicolumn{3}{|c|}{ Widgets } \\
\hline & \multicolumn{3}{|c|}{ Rendering } \\
\hline & Animation & Painting & Gestures \\
\hline & \multicolumn{3}{|c|}{ Foundation } \\
\hline \multirow[t]{4}{*}{$\begin{array}{l}\text { Engine } \\
\mathrm{C} / \mathrm{C}_{++}\end{array}$} & Service Protocol & Composition & Platform Channels \\
\hline & Dart Isolate Setup & Rendering & System Events \\
\hline & Dart Runtime Mgmt & Frame Scheduling & Asset Resolution \\
\hline & & Frame Pipelining & Text Layout \\
\hline \multirow[t]{2}{*}{$\begin{array}{l}\text { Embedder } \\
\text { Platform-specific }\end{array}$} & Render Surface Setup & Native Plugins & App Packaging \\
\hline & Thread Setup & Event Loop Interop & \\
\hline
\end{tabular}


Figure 3. : Flutter framework architecture

In this rendering layer it gives an abstraction which deals with all layouts in this layer that allows us to build a tree of render-able objects. We may also manipulate those objects automatically. The Composition abstraction is provided by the widgets layer. In the rendering layer each rendering object has a corresponding class inside the widgets layer. And also the widgets layer allows us to explain to us about the combination of classes that we can use again.

\section{Case Study}

As we applied a random forest algorithm like a binary tree after the training based on machine learning technique to predict the yields of four crops such as wheat, maize, tomato and potato. To train random forest subtrees, many classification and regression sub-trees are built with the random subset of predictors without cutting them and the forest is averaged. The Source Data for training the model are bootstrapped to generate a random large number of trees. Evaluation of predictor variables is done by how often they make successful predictions or how much they decrease node impurity when they are selected for the splits [30].

\section{Future Challenges}

The Major Challenges that can be encountered in future include population growth, climatic changes, urbanization, increasing completion of natural resources, herbicide resistance, emerging pests and diseases, changing food habits, food losses and wastage. In tropical regions, the challenge is to adapt to the modes of production to the climatic variance by trying to reduce the risks and vulnerability of the production systems and also it is necessary to minimize the pessimistic impacts of agriculture in the environment otherwise there is a chance of increasing the probability of having adverse events. The Minimum Losses from the adverse events can be obtained only by developing the risk indicators which allows anticipations of the impact of threats that must be accompanied by an efficient communication mechanism with a significant scope. So, the internal planning of the production units must be robust in order to implement the conservative techniques, diverse production activities and technical adoption, but for this there should be agricultural services institutions. Since the tropical environment is favorable for continual issues with pests, weeds and diseases. To avoid the calamities from these issues, the crop food animal system needs to be aligned with utilizing biodiverse systems.

With advancements in machine learning and other techniques and methods, building new applications is conceptualized. But the challenge in the technical field is itself that the product of the institution's innovation as one of the rising demands for environmental absorption of residuals is derived from the growth that is commodity production and the consumption. Some of the important concepts and the innovative research ideas are catalogued below:

1. There is a need in developing multidimensional ideas and methods based on conditions like science, industry, expertise and experience.

2. The Introduction of systems that can withstand various types of soils and environmental conditions. As the agricultural lands are harsh and keep on changing either based on the climatic conditions or seasons prevailing.

3. The Development of essential systems that are robust and sustainable to the changes in the external as well as internal factors.

4. The Development of various sensor based systems that are proved to be efficient for highly elevated areas that can mostly have oscillating surfaces and also in most countries it is still a dream to supply power to such areas.

5. The Consumption of past and present data for developing the conclusion taking in support systems for agriculture. However generally the designing of past farming architecture, the important data is only taken into count.

Recent works on Random Forest and CNN algorithms for Crop Protection are highlighted in Table 4.

Table 4: Recent works on Random Forest Algorithm algorithm.

\begin{tabular}{|l|l|l|}
\hline Reference of Proposed Works & Dedicated task of a Crop protection & Main Contributions \\
\hline $\begin{array}{l}\text { Zhang S Ji C, Xu A, Shi Y, and Duan Y } \\
\text { [5] }\end{array}$ & $\begin{array}{l}\text { 3D convolutional neural networks for } \\
\text { crop classification }\end{array}$ & $\begin{array}{l}\text { An effective method achieve } \\
\text { high accuracy in land cover } \\
\text { classification. }\end{array}$ \\
\hline Chouhan S S, Singh U P, and Jain S[9] & $\begin{array}{l}\text { Applications of computer vision in plant } \\
\text { pathology }\end{array}$ & $\begin{array}{l}\text { To develop an appropriate and } \\
\text { espousing a suitable system } \\
\text { for an early and cost-effective } \\
\text { solution for analysing the } \\
\text { prediction. }\end{array}$ \\
\hline Charania I and Li X[11] & Agriculture's shift from a labor intensive \\
\hline
\end{tabular}




\begin{tabular}{|l|l|l|}
\hline \hline & & and robotic techniques. \\
\hline $\begin{array}{l}\text { Puthumalar S,.Ramanujam E, } \\
\text { HariniRajashree R, Kavya C[51] }\end{array}$ & Crop recommendation system & $\begin{array}{l}\text { The proposal aims to achieve } \\
\text { higher accuracy and } \\
\text { performance for Crop } \\
\text { Protection. }\end{array}$ \\
\hline
\end{tabular}

\section{Conclusion}

By summarizing, the organic agricultural practices are a promising method that will hopefully be expanded in the near future. The benefits of buying, eating and producing organically well outweighs the negatives. Even though the cost may be a factor in deciding to buy organic, the health of the soil is more important. In an environmentally conscious world, people need to be more informed about how their food is produced and what effects that is having on the earth and on the future.

\section{References}

1. P. R. Harshani, T. Umamaheswari, R. Tharani, S. Rajalakshmi, and J. Dharani, "Effective crop productivity and nutrient level monitoring in agriculture soil using IOT,'” in Proc. Int. Conf. Soft-Comput. Netw.Secur. (ICSNS), Feb. 2018, pp. 1-10.

2. S. Trilles, J. Torres-Sospedra, Ó. Belmonte, F. J. Zarazaga-Soria, A. González-Pérez, and J. Huerta, " Development of an open sensorized platform in a smart agriculture context: A vineyard support system for monitoring mildew disease," Sustain. Comput.,Informat. Syst., Jan. 2019.

3. S. AashaNandhini, R. Hemalatha, S. Radha, and K. Indumathi, "Web enabled plant disease detection system for agricultural applications using WMSN,’' Wireless Pers. Commun., vol. 102, no. 2, pp. 725740 .

4. S. S. Patil and S. A. Thorat, "Early detection of grapes diseases using machine learning and IoT," in Proc. 2nd Int. Conf. Cognit.Comput. Inf. Process. (CCIP), Aug. 2016, pp. 1-5.

5. S. Ji, C. Zhang, A. Xu, Y. Shi, and Y. Duan, “3D convolutional neural networks for crop classification with multi-temporal remote sensing images,'” Remote Sens., vol. 10, no. 2, p. 75, Jan. 2018, doi: 10. 3390/rs10010075

6. J. Bauer and N. Aschenbruck, "Design and implementation of an agricultural monitoring system for smart farming,"' in Proc. IoT Vertical Topical Summit Agriculture.-Tuscany (IOT Tuscany), Tuscany, Italy, May 2018, pp. 1-6.

7. Murugesan, M., Thilagamani, S. ," Efficient anomaly detection in surveillance videos based on multi layer perception recurrent neural network", Journal of Microprocessors and Microsystems, Volume 79, Issue November 2020, https://doi.org/10.1016/j.micpro.2020.103303

8. G. Zavatta, Agriculture Remains Central to the World Economy. 60\% of the Population Depends on Agriculture for Survival. NetExpo.Accessed: Nov. 9, 2014. http://www.expo2015.org/ magazine/en/economy/agricultureremains-central-to-the-worldeconomy.html

9. S. S. Chouhan, U. P. Singh, and S. Jain, "Applications of computer vision in plant pathology: A survey," Arch. Comput. Methods Eng., vol. 27, no. 2, pp. 611-632, Apr. 2020.

10. Thilagamani, S., Nandhakumar, C. ." Implementing green revolution for organic plant forming using KNN-classification technique", International Journal of Advanced Science and Technology, Volume 29 , Isuue 7S, pp. 1707-1712

I. Charania and X. Li, "Smart farming: Agriculture's shift from a labor intensive to technology native industry,', Internet Things, vol. 9, Mar. 2020, Art. no. 100142.

11. U. Shafi, R. Mumtaz, J. García-Nieto, S. A. Hassan, S. A. R. Zaidi, and N. Iqbal, 'Precision agriculture techniques and practices: From considerations to applications,', Sensors, vol. 19, no. 17, p. 3796, Sep. 2019.

12. Q. Wu, C. Zhao, Y. Liang, D. Zhang, and J. Hao, “Design of farmland information acquisition system based on LoRa wireless sensor network," in Computer and Computing Technologies in Agriculture XI. CCTA (IFIP Advances in Information and Communication Technology), vol. 545. Cham, Switzerland: Springer, 2019.

13. G. Pajares, A. Peruzzi, and P. Gonzalez-de-Santos, "Sensors in agriculture and forestry,' Sensors, vol. 13, no. 9, pp. 12132-12139, Sep. 2013.

14. Thilagamani, S., Shanti, N.," Gaussian and gabor filter approach for object segmentation", Journal of Computing and Information Science in Engineering, 2014, 14(2), 021006, https://doi.org/10.1115/1.4026458 
15. Aqeelur-Rehman, A. Z. Abbasi, N. Islam, and Z. A. Shaikh, "A review of wireless sensors and networks' applications in agriculture,' Comput. Standards Interface, vol. 36, no. 2, pp. 263-270, Feb. 2014.

16. R. Chokkareddy, N. Thondavada, and S. Kanchi, "Recent trends in sensors for health and agricultural applications,' in Advanced Biosensors for Health Care Applications. Amsterdam, The Netherlands: Elsevier, 2019, pp. 341-355.

17. FarmBeats. Accessed: 2015 , https:// www.microsoft.com/en-us/research/project/farmbeats-iotagriculture/

18. Rhagini, A., Thilagamani, S. ,'Women defence system for detecting interpersonal crimes",International Journal of Advanced Science and Technology, 2020, Volume 29,Issue7S, pp. 1669-1675

19. Santhi, P., Priyanka, T.,Smart India agricultural information reterival system, International Journal of Advanced Science and Technology, 2020, 29(7 Special Issue), pp. 1169-1175.

20. Environmental Factors Affecting Plant Productivity, Matshal A Haferkamp, Fort Keogh Research Symposium,Sep 1987

21. Plant growth and soil moisture relationships, J.FBierhuizen, Unesco/NS/AZ/476, Madrid Symposium Paper N0.37, Paris 1 September 1959

22. Santhi, P., Mahalakshmi, G., Classification of magnetic resonance images using eight directions gray level co-occurrence matrix $(8 \mathrm{dglcm})$ based feature extraction, International Journal of Engineering and Advanced Technology, 2019, 8(4), pp. 839-846

23. Influence of Autumn Tilth on Soil Physical Conditions andTiming of Crop Establishment in Spring J. agric. Engng Res. (1984) 29, 265-213

24. Salinity and irrigation method affect crop yield and soil Quality In watermelon growing, Published online 20 February 2008 in Wiley Inter Science .www.interscience.wiley.com

25. Deepa, K., Kokila, M., Nandhini, A., Pavethra, A., Umadevi, M. "Rainfall prediction using CNN", International Journal of Advanced Science and Technology, 2020, 29(7 Special Issue), pp. 1623-1627. http://sersc.org/journals/index.php/IJAST/article/view/10849.

26. D. Coomans; D.L. Massart (1982). "Alternative k-nearest neighbor rules in supervised pattern recognition: Part 1. K-Nearest neighbor classification by using alternative voting rules".AnalyticaChimica Acta.136: 15-27.

27. P. Pandiaraja, N Deepa 2019," A Novel Data Privacy-Preserving Protocol for Multi-data Users by using genetic algorithm", Journal of Soft Computing, Springer, Volume 23 ,Issue 18, Pages 8539-8553,

28. Breiman, L., Friedman, J.H., Olshen, R.A., Stone, C.J., 1984. ClassificationAnd Regression Trees. Wadsworth.

29. N Deepa , P. Pandiaraja, 2020 ," Hybrid Context Aware Recommendation System for E-Health Care by merkle hash tree from cloud using evolutionary algorithm" , Journal of Soft Computing, Springer , Volume 24 ,Issue 10, Pages 7149-7161

30. K Sumathi, P Pandiaraja 2019," Dynamic alternate buffer switching and congestion control in wireless multimedia sensor networks", Journal of Peer-to-Peer Networking and Applications, Springer, Volume 13,Issue 6,Pages 2001-2010

31. Neural Network Applications In Agricultural Economics Jianhua Chen

32. Biom. J. 34 (1992) 4, 501-511 AkademieVerlag Probability Model for Crop Yield ForecastingR. C. JAIN and RANJANG UWALI.A.S.R.I., New Delhi

33. Pandiaraja, P, Vijayakumar, P, Vijayakumar, V \& Seshadhri, R 2017, 'Computation Efficient Attribute Based Broadcast Group Key Management for Secure Document Access in Public Cloud', Journal of Information Science and Engineering, 33, No. 3, pp. 695-712.

34. Veenadhari, S. Misra, Bharat; Singh, CD -- [IEEE 2014 International Conference on Computer Communication and Informatics (ICCCI) - Coimbatore, India (2014.1.3-2014.)

35. N Deepa , P. Pandiaraja, 2020, “E health care data privacy preserving efficient file retrieval from the cloud service provider using attribute based file encryption “, Journal of Ambient Intelligence and Humanized Computing, Springer, https://doi.org/10.1007/s12652-020-01911-5

36. Kumar, Rakesh; Singh, M.P.; Kumar, Prabhat; Singh, J.P. -- [IEEE 2015 International Conference on Smart Technologies and Â Management Â forÂ Computing, Communication

37. Dimitriadis, Savvas; Gounopoulos, Christos -- [IEEE 2008 12th Panhellenic Conference on Informatics - Samos, Greece (2008.08.28-2008.08.30)]

38. Vijayakumar, P, Pandiaraja, P, Balamurugan, B \& Karuppiah, M 2019, 'A Novel Performance enhancing Task Scheduling Algorithm for Cloud based E-Health Environment', International Journal of E-Health and Medical Communications, Vol 10,Issue 2,pp 102-117. 
39. MachineLearning: What it is and why it matters, 09 2016, www.sas.com

40. R. E. Schapire, "The boosting approach to machine learning: An overview" in Nonlinear estimation and classification, New York:Springer, pp. 149-171, 2003.

41. Mukhopadhyay S.C. (2012) Smart Sensing Technology for Agriculture and Environmental Monitoring. Vol. 146, Springer Berlin Heidelberg.

42. Jun Wu, AnastasiyaOlesnikova, Chi-Hwa Song, Won Don Lee (2009). The Development and Application of Decision Tree for Agriculture Data. IITSI :16-20.

43. Leemans, V., Destain, M.F.,2004.A real-time grading method of apples based on features extracted from defects. J. Food Eng. 61, 83-89.

44. Quinlan, J.R.(1985b). Decision trees and multi-valued attributes. In J.E. Hayes \& D. Michie (Eds.), Machine intelligence 11. Oxford University Press (in press).

45. Y. Hayami, V. W. Ruttan, Agricultural development: an international perspective, Baltimore, London:The Johns Hopkins Press, 1971.

46. S. Veenadhari, D. Bharat Mishra, D. C. Singh, "Soybean Productivity Modelling Using Decision Tree Algorithms", International Journal of Computer Applications, vol. 27, no. 7, pp. 11-15, Aug. 2011.

47. M. S. Dahikar, D. V. Rode, "Agricultural Crop Yield Prediction Using Artificial Neural Network Approach", INTERNATIONAL JOURNAL OF INNOVATIVE RESEARCH IN ELECTRICAL ELECTRONICS INSTRUMENTATION AND CONTROL ENGINEERING, vol. 2, no. 1, pp. 683-686, 2014.

48. M. Somvanshi, P. Chavan, "A review of machine learning techniques using decision tree and support vector machines", 2016 International Conference on Computing Communication Control and automation (ICCUBEA), pp. 1-7, 2016.

49. German, L., Ramisch, J.J. \&Verma R. (2010) Beyond the Biophysical, Knowledge, Culture, and Power in Agriculture and Natural Resource Management, Springer Publ.

50. S.Pudumalar, E. Ramanujam, R. HarineRajashree, C. Kavya,"'Crop recommendation system for precision agriculture" 